\title{
Evaluation of Skin Barrier Function Using Direct Current II: Effects of Duty Cycle, Waveform, Frequency and Mode
}

\author{
Makoto KanebaKo, ${ }^{*}, a$ Toshio Inagi, ${ }^{a}$ and Kozo TAKayAma ${ }^{b}$ \\ ${ }^{a}$ Fuji Research Laboratories, Pharmaceutical Division, Kowa Company, LTD.; 332-1 Ohnoshinden, Fuji, Shizuoka \\ 417-8650, Japan: and ${ }^{b}$ Department of Pharmaceutics, Hoshi University; 2-4-41 Ebara, Shinagawa-ku, Tokyo 142-8501, \\ Japan. Received July 8, 2002; accepted September 27, 2002
}

\begin{abstract}
The aim of this study was to evaluate the reduction in skin barrier function caused by pulsed iontophoresis by measuring resistance in the short term. Experiments under direct current (DC) and pulsed direct current (PDC) conditions were carried out using rat abdominal skin in vivo. The resistance was measured every $62.5 \mu \mathrm{s}$ and analyzed using a two-compartment model consisting of surface and skin resistance. Moreover, the initial value and the rate constant of surface resistance were calculated with the non-linear approximation program. Using this method, effects of duty cycle, waveform, frequency and mode on the skin barrier function were examined. The barrier function decreased with increasing duty cycle. With regard to waveforms, the influence on the skin barrier function was greatest in the order of sine, rectangular, saw and triangular waveforms. A frequency of less than $100 \mathrm{~Hz}$ reduced the barrier function. Considering the reduction in barrier function and skin polarization, the PDC of a rectangular waveform (around $75 \%$ as duty cycle and less than $100 \mathrm{~Hz}$ as frequency) or sine waveform was most suitable. In addition, the difference in the amount of drug delivered by DC and by PDC was discussed.
\end{abstract}

Key words pulsed iontophoresis; skin barrier function; duty cycle; waveform; frequency; mode

The transdermal delivery of a drug has several advantages including the avoidance of a first-pass effect, avoidance of side effects and expression of a topical drug effect. However, disadvantages include a low absorption of high molecular weight and hydrophilic compounds, and enhancement of the amount of drug absorbed.

To overcome these problems, we have focused on iontophoresis, one of several physical methods. In previous studies, we predicted that the migration of a drug by iontophoresis was the result of a synergistic effect of current application and enhanced passive diffusion. ${ }^{1)}$ The prediction was proved valid by measuring the skin distribution of indomethacin using a cryostat. ${ }^{2)}$ In that study, the permeation from the stratum corneum to upper dermis was increased by iontophoresis. The increase in the stratum corneum depended on a reduction in the skin barrier function. On the other hand, the increase from the epidermis to upper dermis contributed to the passage of current. Furthermore, we proposed a new method of analyzing skin barrier function, the measurement of resistance in the short term. ${ }^{3)}$ The resistance was taken as a two-compartment model, which consisted of the sum of the surface compartment (i.e., surface resistance) and the skin compartment (i.e., skin resistance). In addition, the initial value and the rate constant, which were calculated with a non-linear approximation program using a two-compartment model, could evaluate the reduction in skin barrier function. It is important to evaluate the barrier function in the short term because the resistance drops remarkably in that time and gradually thereafter when a direct current is applied.

When a current is applied directly to the skin, polarization and irritation occur immediately. ${ }^{4)}$ To avoid this, the application of pulsed direct current (PDC) has been examined. Numajiri et al. compared the percutaneous absorption of sodium benzoate between direct current (DC) $\left(0.1 \mathrm{~mA} / \mathrm{cm}^{2}\right)$ and PDC $\left(3 \mathrm{~mA} / \mathrm{cm}^{2}\right.$, rectangular waveform, $40 \mathrm{kHz}$ and $30 \%$ duty cycle). ${ }^{5)}$ They reported that the amount of drug absorbed was almost equal and PDC was more suitable in terms of skin irritation. Zakzewski et al. ${ }^{\text {()) }}$ and Bagniefski et $a l^{7)}$ also showed an equivalent absorption between DC and PDC. Meanwhile, Preat et al. reported that the absorption by DC $\left(0.17 \mathrm{~mA} / \mathrm{cm}^{2}\right)$ was greater than that by PDC $\left(0.17 \mathrm{~mA} / \mathrm{cm}^{2}\right.$, rectangular waveform, $2500 \mathrm{~Hz}$ and $50 \%$ duty cycle) using sufentanil citrate. ${ }^{8)}$ Conversely, Huang and $\mathrm{Wu}$ suggested that the absorption was greater by PDC ( 0.64 $\mathrm{mA} / \mathrm{cm}^{2}$, rectangular waveform, $3 \mathrm{kHz}$ and $50 \%$ duty cycle) than $\mathrm{DC}\left(0.32 \mathrm{~mA} / \mathrm{cm}^{2}\right)$ using thyrotropin-releasing hormone (TRH). ${ }^{9)}$ A similar tendency was reported by Knoblauch and Moll using buserelin between PDC $\left(0.1-0.2 \mathrm{~mA} / \mathrm{cm}^{2}\right.$, rectangular waveform, $2 \mathrm{kHz}$ and $50 \%$ duty cycle) and DC (0.1 $\left.\mathrm{mA} / \mathrm{cm}^{2}\right)^{10)}$

As mentioned above, the absorption of drug by DC and PDC iontophoresis has differed with investigators. This can be attributed to the different current application conditions and poor clarification of the relationship between pulsed iontophoresis and the reduction in skin barrier function. The amount of drug that migrated by iontophoresis was the sum of the amount moved by passive diffusion, current application and electroosmosis. ${ }^{11-13)}$ Furthermore, the amount due to passive diffusion was closely related to the reduction in skin barrier function. ${ }^{1)}$ Thus, the reduction in barrier function caused by pulsed iontophoresis is important to clarifying the migration of a drug.

The aim of this study was to evaluate the barrier to pulsed iontophoresis by measuring resistance in the short term. ${ }^{3)}$ In detail, the effects of duty cycle, waveform, frequency and mode on barrier function were examined.

\section{MATERIALS AND METHODS}

Materials and Animals $N$-Vinyl acetamide sodium acrylate copolymer (PNVA) was purchased from Showa Denko K. K. (Tokyo, Japan). The PNVA was a white powder with molecular weights of about 2300000 (number-average molecular weight; Mn), 2600000 (weight-average molecular 
weight; Mw) and 3000000 (z-average molecular weight; $\mathrm{Mz}$ ). All other chemicals were of analytical grade obtained from dealers. Silver and silver chloride electrodes (Ionview) were bought from ADVANCE Co., Ltd. (Tokyo, Japan). Male Wistar rats ( 8 weeks old, $200 \mathrm{~g}$ ) were purchased from Charles River Japan, Inc. (Tokyo, Japan).

Preparation of Adhesive Pads Sodium chloride (0.05, $0.1,0.2 \mathrm{~g})$, tartaric acid $(0.2 \mathrm{~g})$ and carrageenan $(0.5 \mathrm{~g})$ were dissolved in purified water $(50 \mathrm{~g})$ at $50^{\circ} \mathrm{C}$ (water phase). Meanwhile, anhydrous aluminum hydroxide gel $(0.4 \mathrm{~g})$, hydroxypropylcellulose $(3 \mathrm{~g})$ and PNVA $(10 \mathrm{~g})$ were suspended in macrogol $400(30 \mathrm{~g})$ at room temperature (oil phase). After the water phase had cooled to room temperature, the oil phase was added. Total weight was adjusted to $100 \mathrm{~g}$ by adding purified water. The mixture was shaken using a mixer (HIVISMIX, Tokushukika Kogyo Co., Ltd., Osaka, Japan) at a combination of $40 \mathrm{rpm}$ (revolution) and $80 \mathrm{rpm}$ (rotation) for $10 \mathrm{~min}$. The mixture was rolled $750 \mu \mathrm{m}$ thick between polyethylene liners, the surface of which was processed with silicone, using a roller machine (Ikeda Machine Industry Co., Ltd., Osaka, Japan). After the rolling, the liner on one side was peeled away. The silver and silver chloride electrodes were then installed on the mixture and the mixture cut circularly $(30 \mathrm{~mm} \phi)$ with the electrode and liner as shown in Fig. 1. Thus, the adhesive pads were prepared.

Measurement of Resistance in Vivo The rats were anesthetized with an intraperitoneal injection of pentobarbital $(0.05 \mathrm{mg} / \mathrm{g})$ and their abdominal hair was clipped. Two adhesive pads were applied to the abdominal skin $1 \mathrm{~cm}$ apart. The $\mathrm{Ag}$ (anode) and $\mathrm{AgCl}$ (cathode) electrodes were connected to a function generator (WAVE FACTORY Type 1496, NF ELECTRONIC INSTRUMENTS, Kanagawa, Japan) as shown in Fig. 2. The experiments were carried out under DC and PDC conditions. The voltage and current were measured with a oscillographic recorder (Type OR-100, Yokogawa Electric Co., Ltd., Tokyo, Japan) at intervals of $62.5 \mu$ s for $8 \mathrm{~s}$. The data were transformed to Microsoft Excell 97 for windows using the Handy OR Application (Yokogawa Electric Co., Ltd., Tokyo, Japan). Each resistance at intervals of $62.5 \mu \mathrm{s}$ was calculated according to Ohm's law using Microsoft Excell 97 for windows. The above mentioned timeresistance data were fitted to an equation which represented the sum of the exponential function of the surface and skin resistance. ${ }^{3)}$ The fitting program used was a non-linear approximation program, MULTI. Furthermore, the initial value and the rate constant of the surface resistance were calculated.

\section{RESULTS AND DISCUSSION}

Taking into consideration the pulsed iontophoresis, the influence of duty cycle, waveform, frequency and mode on the skin barrier function was significant. We investigated the effect of these factors using the method proposed in a previous study. ${ }^{3)}$ The experiments under DC and PDC conditions were performed with rat abdominal skin in vivo. A constant voltage was used because the proposed method was based on such conditions. Moreover, to clarify the tracking of current and voltage, measurements were carried out every $62.5 \mu \mathrm{s}$.

Effect of Duty Cycle Nakakura et al. reported that when a pulsed current $\left(0.2 \mathrm{~mA} / \mathrm{cm}^{2}\right.$, rectangular waveform, $2 \mathrm{kHz}$

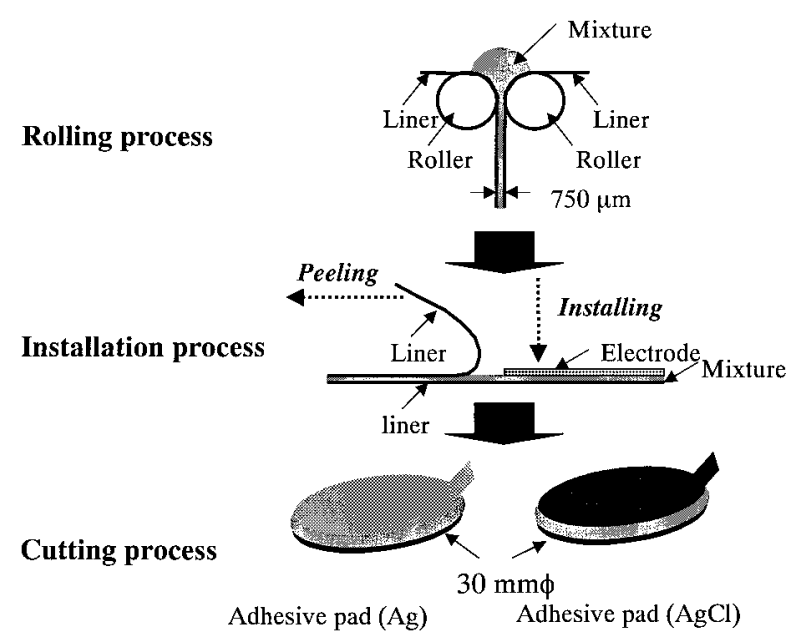

Fig. 1. The Preparation of the Adhesive Pad

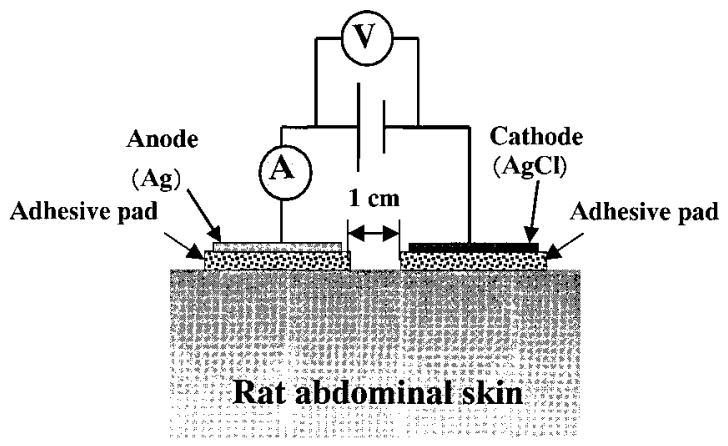

Fig. 2. The Experimental Set-up for Measuring Resistance in Vivo A, oscillographic recorder; $\mathrm{V}$, function generator.

and $26-100 \%$ duty cycle) was applied, the initial voltage increased with increasing duty cycle. ${ }^{14)}$ In other words, the resistance depended on the duty cycle. In contrast, Chen and Chien, ${ }^{15)}$ Chien et al. ${ }^{16)}$ and Nakakura et $a l .{ }^{14)}$ showed that the duty cycle contributed to the percutaneous absorption of a drug. To clarify the effect of duty cycle on barrier function, the proposed method was used.

The experiments were carried out at $6 \mathrm{~V}$ with a rectangular waveform, $1 \mathrm{kHz}$ frequency and $3 \mathrm{~V}$ offset. The voltage and current profiles for $2 \mathrm{~ms}$ immediately and $8 \mathrm{~s}$ after the application of voltage are shown in Fig. 3. Although the voltage was constant $(6 \mathrm{~V})$, the initial resistance differed with duty cycle. This is the reason why the initial resistance immediately after the application of direct current could not be measured accurately. The resistance changed remarkably due to the occurrence of skin polarization. The measurement time of $8 \mathrm{~s}$ was selected because the resistance was almost in equilibrium at that time. Immediately after the application of current, current and voltage tracked from a 25 to $100 \%$ duty cycle were weak until $0.5 \mathrm{~ms}$. However, they improved with time as shown in Fig. 3a. Moreover, an increase in current, i.e., reduction in resistance, was observed with increasing duty cycle. At $8 \mathrm{~s}$ after the application of current, the tracking of current and voltage of all duty cycles improved compared to that immediately after the application of voltage as shown in Fig. 3b. Skin polarization, which was a reduction of current during the pulse application, was recognized at less than a $90 \%$ duty cycle. At a $100 \%$ duty cycle, i.e., direct cur- 
(a)
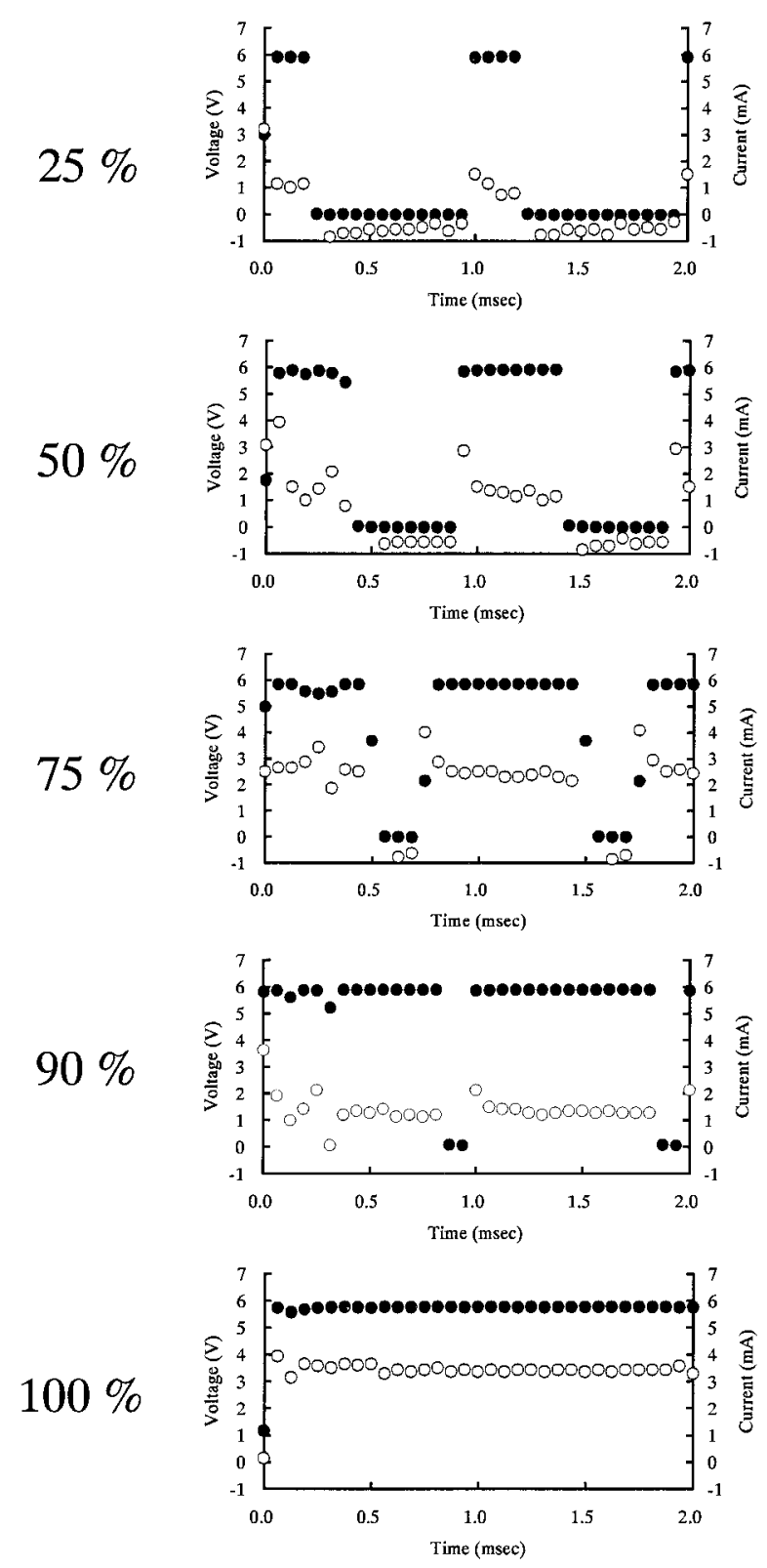

(b)
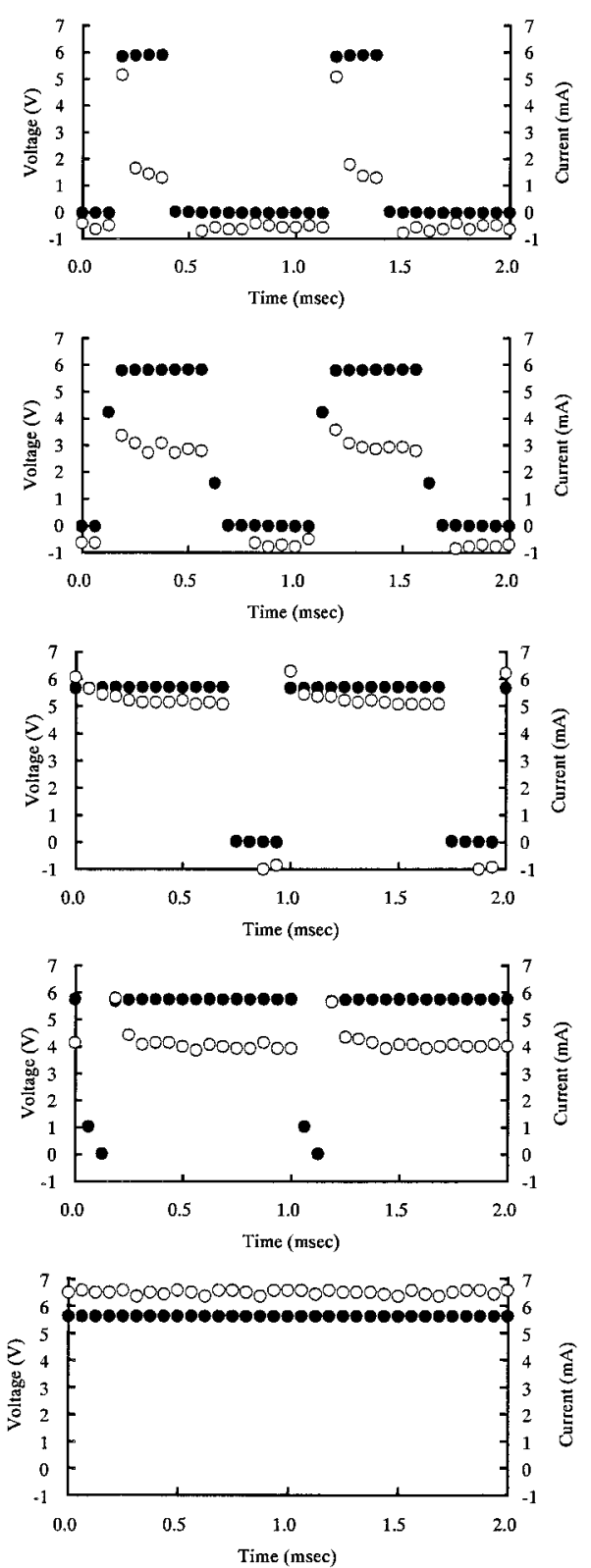

Fig. 3. Effect of Duty Cycle (6 V, Rectangular Waveform, $1 \mathrm{kHz}, 3 \mathrm{~V}$ Offset) on the Voltage (O) and Current (O) Profiles Immediately (a) and $8 \mathrm{~s}(\mathrm{~b})$ after the Application of Voltage

Each datum is the mean $(n=3)$.

rent, the current was almost constant since skin depolarization did not occur again. Furthermore, a depolarization, i.e., a higher current at about $1.19 \mathrm{~ms}$ than $0.48 \mathrm{~ms}$ in Fig. $3 b$ (25\%), was also observed. These phenomena improved with time. It was thought that the low-level tracking of the current and voltage immediately after the application of current depended on the structural change to skin. Chizmadzhev et $a l .{ }^{17)}$ reported that current passed through the lipid-corneocyte matrix (e.g., lipid bilayer) and skin appendages (e.q., sweat glands and hair follicles). The lipid-corneocyte matrix formed a pore when the current was applied. This is why the skin barrier function decreases and the drug penetrates the skin easily. Therefore, immediately after the application of current, it was assumed that the tracking of current and volt- age were poor since a pore had formed. The tracking improved with time because the pore size becomes constant. Similar results were reported by Hirvonen et al. ${ }^{18)}$ They guessed at the behavior from the voltage delay one hour after the application of current $\left(0.25 \mathrm{~mA} / \mathrm{cm}^{2}\right.$, rectangular waveform, $25-2500 \mathrm{~Hz}$ and $50 \%$ duty cycle).

Resistance-time profiles are shown in Fig. 4. The resistance immediately after the application of voltage decreased and the damping increased with increasing duty cycle. These results contributed to the voltage application time and total current. To clarify the change mentioned above, the initial value and the rate constant of the surface resistance were calculated using our proposed method (Fig. 5). ${ }^{3)}$ The initial value evidently decreased until a $75 \%$ duty cycle, and did not 


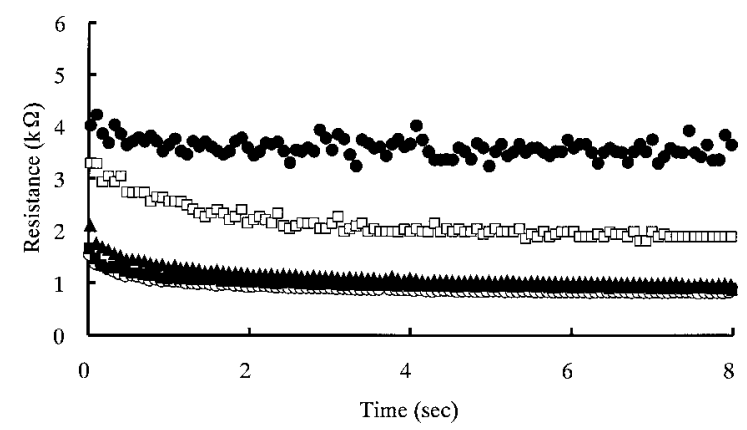

Fig. 4. Effect of Duty Cycle (6 V, Rectangular Waveform, 1 kHz, 3 V Offset) on Resistance-Time Profiles for $8 \mathrm{~s}$

- $25 \% ; \square, 50 \% ; \boldsymbol{\Delta}, 75 \% ; \bigcirc, 90 \% ; \square, 100 \%$. Each datum is the mean $(n=3)$.

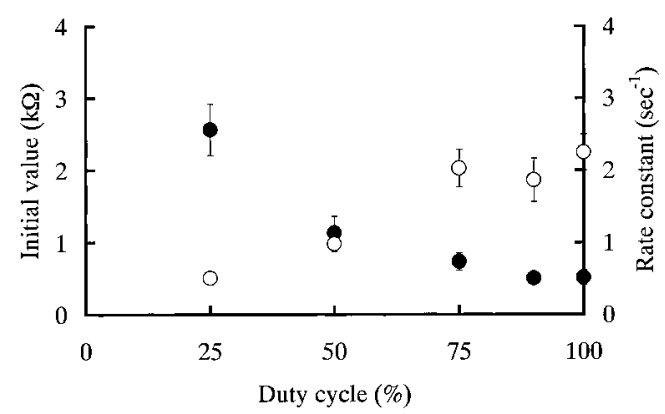

Fig. 5. Effect of Duty Cycle (6 V, Rectangular Waveform, 1 kHz, 3 V Offset) on the Initial Value $(O)$ and the Rate Constant $(\bigcirc)$ of Surface Resistance

Each datum is the mean \pm S.E. $(n=3)$.

decrease thereafter. In contrast, the rate constant increased until a $75 \%$ duty cycle, and did not increase thereafter. These results showed the reduction in skin barrier function by the application of PDC with a $75 \%$ duty cycle in addition to that by DC. Thus, the use of PDC with a duty cycle of around $75 \%$ was suitable because skin polarization occurred when DC was applied. As regards the absorption of drug, it was enhanced by increasing duty cycle due to the reduction in skin barrier function in addition to the increase in total current.

Effect of Waveforms Chen and Chien ${ }^{15}$ reported the percutaneous absorption of LHRH using DC $\left(0.764 \mathrm{~mA} / \mathrm{cm}^{2}\right)$ and PDC $\left(0.764 \mathrm{~mA} / \mathrm{cm}^{2}, 1 \mathrm{kHz}\right.$ and $50 \%$ duty cycle $)$ with different waveforms. Although the iontophoretic flux caused by both sine and rectangular waveforms was equal to that for DC, the flux caused by a triangular waveform was less than that for the other waveforms and DC. Chien et al. ${ }^{16)}$ showed that in terms of the reduction in blood glucose level caused by insulin, the waveforms ranked in the order of sine, trapezoidal and rectangular $\left(0.33 \mathrm{~mA} / \mathrm{cm}^{2}, 2 \mathrm{kHz}\right.$ and $50 \%$ duty cycle). In addition, Hirvonen et al. ${ }^{18)}$ reported that the skin permeability of amino acids (lysine and glutamic acid) caused by a rectangular waveform was equal to that due to a sine one $\left(0.5 \mathrm{~mA} / \mathrm{cm}^{2}, 2.5 \mathrm{kHz}\right.$ and $50 \%$ duty cycle). As mentioned above, the waveforms were expected to affect to the permeability of the drug. To clarify this, the same analysis was performed.

The experiments were carried out at $6 \mathrm{~V}$ (voltage), $1 \mathrm{kHz}$ (frequency) and 50\% (duty cycle) using rectangular, sine, saw and triangular waveforms. The voltage and current pro- files for $2 \mathrm{~ms}$ both immediately and $8 \mathrm{~s}$ after the application of voltage are shown in Fig. 6. Immediately after the application of voltage, current and the tracking of voltage and current of the sine waveform were highest as shown in Fig. 6a. At $8 \mathrm{~s}$ after the application of voltage, those of the sine waveform were highest, and those of other waveforms were little improved. Although maximum current was more than $5 \mathrm{~mA}$ for the sine waveform, it was less than $4 \mathrm{~mA}$ for the others. These results suggested that the skin polarization and depolarization did not occur easily with the sine waveform. In addition, the reduction in barrier function was greater for the sine waveform than the other waveforms. A similar tendency was reported by Hirvonen et al., ${ }^{18)}$ who compared the tracking of current and voltage after a one-hour application of current between rectangular and sine waveforms. However, our results reflected the identity of the reduction in barrier function since structural changes to the skin were observed in the short term. The measurement in the short term is important to evaluate the barrier function since it decreased remarkably in that time.

The effects of waveforms on the initial value and the rate constant of surface resistance are shown in Fig. 7. Although the initial values were almost equal, the rate constant was highest in the order of sine, rectangular, saw and triangular waveforms. These results showed that the sine waveform decreased the barrier function most effectively. From the results mentioned above, the effect on the reduction in barrier function of the sine waveform was almost equal to that of the rectangular waveform with a 75\% duty cycle (see Figs. 5, 7). Notably, although the sine waveform was similar to the triangular one, its effect on skin barrier function was significantly grater. It was predicted that the reason for this was the " $1 / \mathrm{f}$ fluctuation". Further investigation is needed to clarify the mechanism involved.

With respect to the relationship between the skin permeability of a drug and waveforms, the results by Chen and Chien ${ }^{15)}($ sine $=$ rectangular $>$ triangle $)$ and by Chien et al. $\left.{ }^{16}\right)$ (sine $>$ trapezoidal $=$ rectangular) were similar to our findings. Thus, waveforms which reduced the barrier function, enhanced the drug delivery into the skin. However, Hirvonen et $a l .{ }^{18)}$ reported that waveforms (sine and rectangular) did not affect the absorption of drug. It was assumed that materials (lysine and glutamic acid) mainly migrated with the current due to a low molecular weight and hydrophilic properties.

Effect of Frequency As regards the contribution of frequency to absorption by pulsed iontophoresis, Chien et al. reported that the absorption of insulin caused by $2 \mathrm{kHz}$ was higher than that caused by $1 \mathrm{kHz} .^{16)}$ On the other hand, Bagniefski et ll ${ }^{7)}$ and Nakakura et $a l .{ }^{14)}$ showed that the effect of frequency on the absorption of the drug was almost unrecognizable. To clarify the effect of frequency on the barrier function, our proposed method was examined.

The experiments were performed at $6 \mathrm{~V}$ with a rectangular waveform, $50 \%$ duty cycle and $3 \mathrm{~V}$ offset. The rectangular waveform was selected to discuss the relationship between frequency and the absorption of the drug in previous studies. $^{7,14,15)}$ Although the initial values were almost the same until $1000 \mathrm{~Hz}$, the rate constant decreased more than $100 \mathrm{~Hz}$ as shown in Fig. 8. These results suggested that the lower frequency (less than $100 \mathrm{~Hz}$ ) was effective in decreasing the barrier function. As for the rectangular waveform, the 
(a)
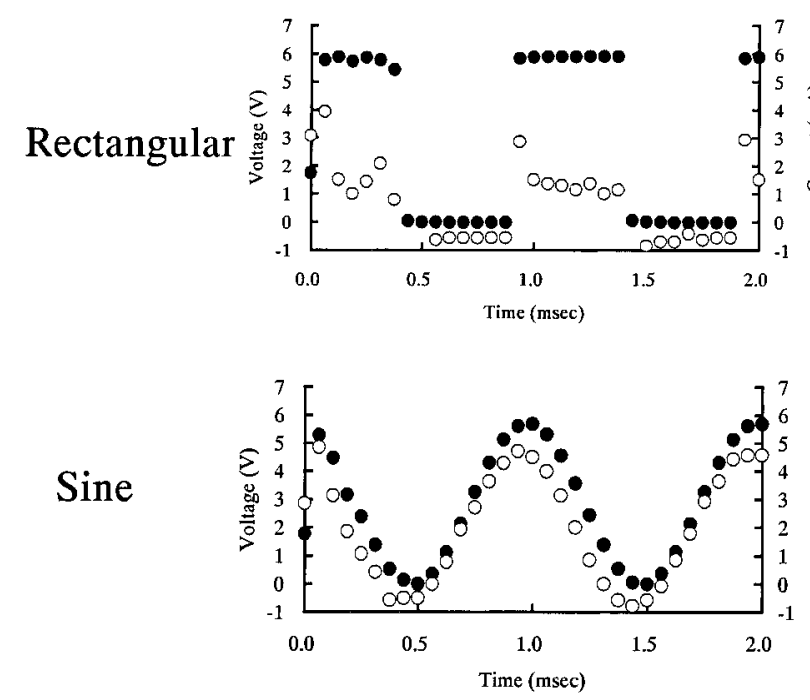

Sine

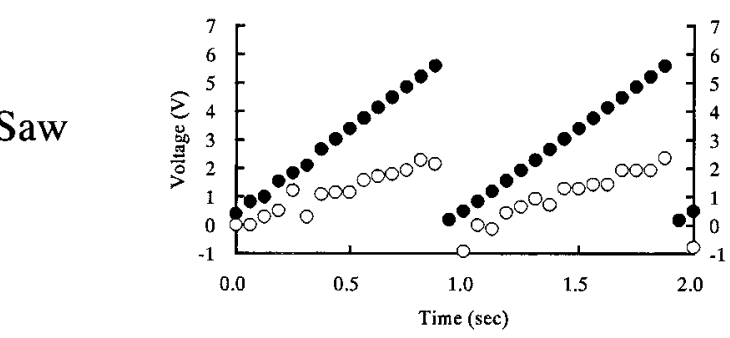

Saw
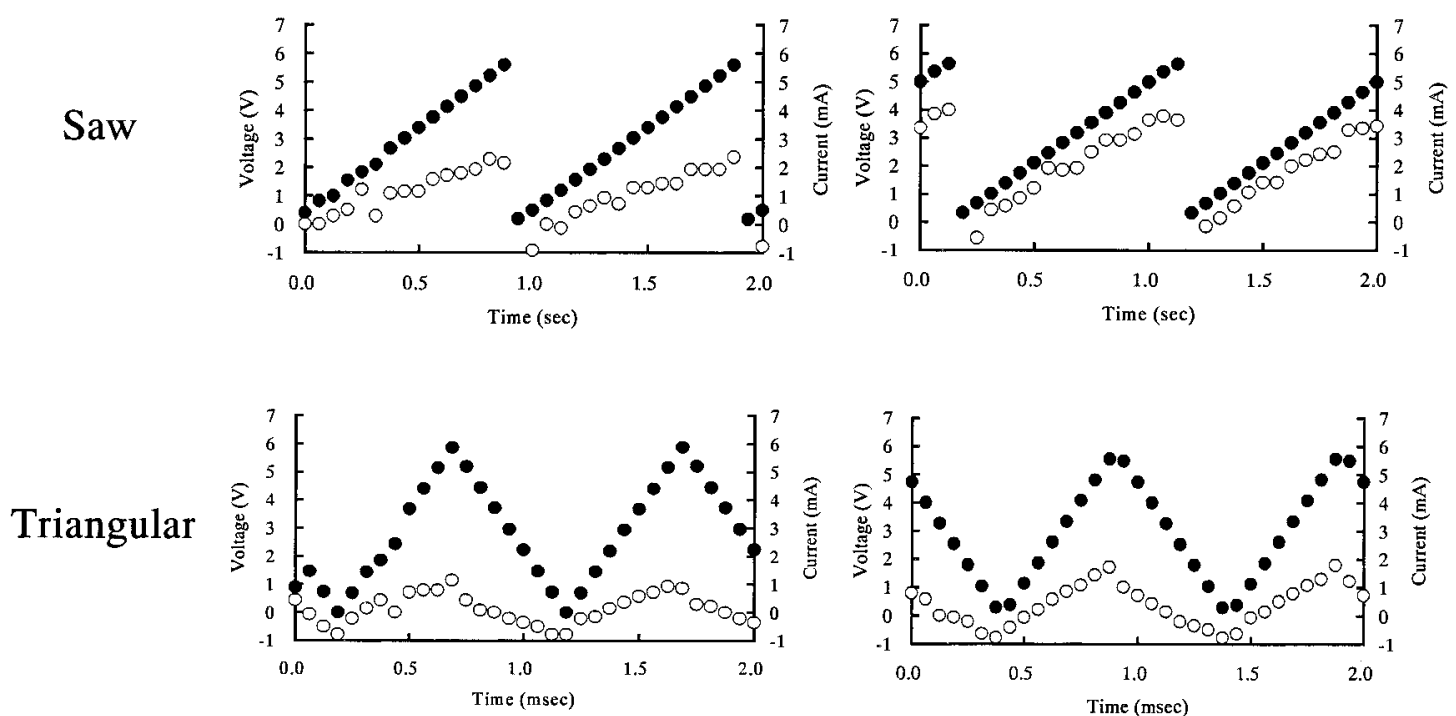

(b)
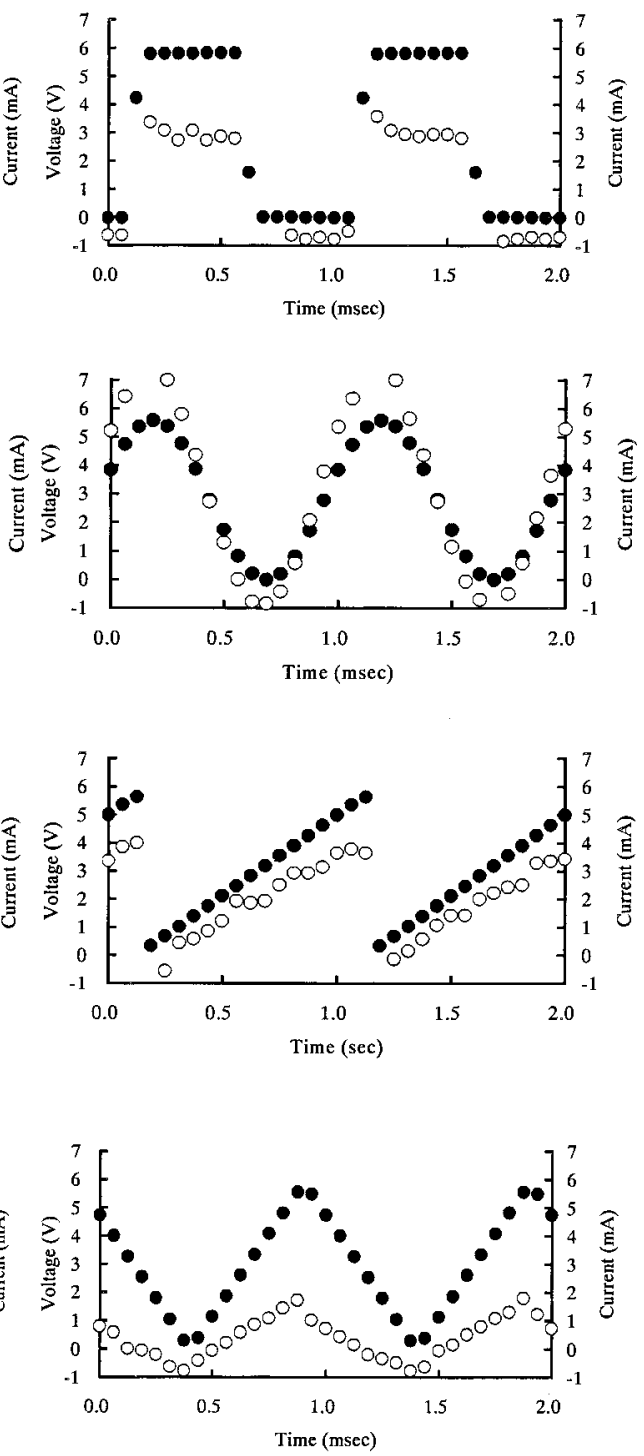

Fig. 6. Effect of Rectangular, Sine, Saw and Triangular Waveforms (6 V, 1 kHz, 50\% Duty Cycle and 3 V Offset) on the Voltage (d) and Current (O) Profiles Immediately (a) and $8 \mathrm{~s}$ (b) after the Application of Voltage

Each datum is the mean $(n=3)$.

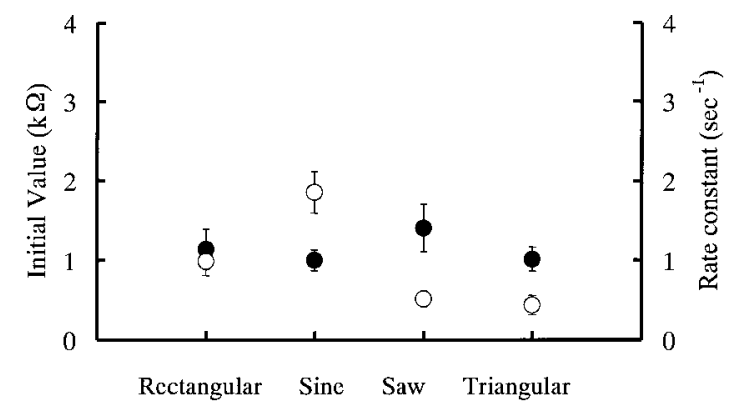

Fig. 7. Effect of Waveforms (6 V, $1 \mathrm{kHz}, 50 \%$ Duty Cycle and $3 \mathrm{~V}$ Offset) on the Initial Value $(\bullet)$ and the Rate Constant $(\bigcirc)$ of Surface Resistance

Each datum is the mean \pm S.E. $(n=3)$.

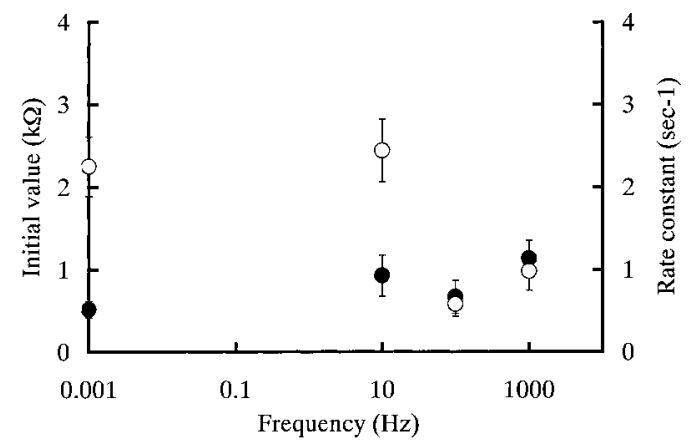

Fig. 8. Effect of Frequency (6 V, Rectangular Waveform, 50\% Duty Cycle and 3 V Offset) on the Initial Value $(-)$ and the Rate Constant $(O)$ of Surface Resistance

Each datum is the mean \pm S.E. $(n=3)$. 
current increased with increasing duty cycle as shown in Fig. 3 . These factors contributed to the pulse length. It was assumed that similar behavior occurred when the frequency increased. Thus, the rate constant decreased with increasing frequency because pulse length shortened. Therefore, a frequency of less than $100 \mathrm{~Hz}$ was suitable to reduce the barrier function.

With respect to the absorption of drug, Bagniefski and Burnette $^{7)}$ and Nakakura et al. $^{14)}$ reported that the frequency did not affect the absorption. It was assumed that the effect of frequency on the barrier function was low because they used more than $1 \mathrm{kHz}$. On the other hand, Chien et al. ${ }^{16)}$ reported that the absorption of insulin caused by $2 \mathrm{kHz}$ was higher than that caused by $1 \mathrm{kHz}$. However, the difference was small.

Effect of Mode For comparison of the absorption of drug between DC and PDC, the results differed with investigators. To clarify the difference, resistance-time profiles for both modes were plotted (Fig. 4). The data used were for DC $(6 \mathrm{~V})$ and PDC (6 V, rectangular waveform, $1 \mathrm{kHz}, 50 \%$ duty cycle and offset $3 \mathrm{~V})$.

The resistance by DC was significantly higher than that by PDC for $8 \mathrm{~s}$. Furthermore, in the evaluation using the proposed method, although the initial value was higher for PDC (50\% duty cycle) than DC (100\% duty cycle), the rate constant was lower with PDC than DC (see Fig. 5). These results suggested that the reduction in skin barrier function caused by DC was higher than that due to PDC at the same voltage. In a previous study, ${ }^{1)}$ we reported that the amount of drug delivered by iontophoresis was the sum of that delivered by current application and by enhanced passive diffusion. In addition, the reduction in skin barrier function depends on voltage. ${ }^{3)}$ Thus, it was assumed that the absorption of drug by DC was greater than that by PDC at the same voltage due to enhanced passive diffusion.

However, Zakzewski et al. $\left.{ }^{6}\right)$ showed that the absorption of captopril by $\mathrm{DC}\left(0.024 \mathrm{~mA} / \mathrm{cm}^{2}\right)$ was almost equal to that by PDC $\left(0.08 \mathrm{~mA} / \mathrm{cm}^{2}\right.$, rectangular waveform, $1 \mathrm{kHz}$ and $30 \%$ duty cycle) using the same total current. This result contributed to almost the same reduction in barrier function because the voltage by PDC was higher than that by DC. In addition, Huang and $\mathrm{Wu}^{9}$ ) reported that the absorption of TRH by PDC $\left(0.64 \mathrm{~mA} / \mathrm{cm}^{2}\right.$, rectangular waveform, $3 \mathrm{kHz}$ and $50 \%$ duty cycle) was higher than that by DC $\left(0.32 \mathrm{~mA} / \mathrm{cm}^{2}\right)$. This was because of the marked reduction in barrier function caused by PDC due to the significantly higher voltage. They performed the experiments with a constant total current in consideration of the duty cycle when PDC was applied. Here, the absorption by enhanced passive diffusion due to the reduction in barrier function was neglected. On the other hand, Preat and Thysman ${ }^{8)}$ showed that the absorption of sufentanil citrate by DC $\left(0.17 \mathrm{~mA} / \mathrm{cm}^{2}\right)$ was higher than that by PDC $\left(0.17 \mathrm{~mA} / \mathrm{cm}^{2}\right.$, rectangular waveform, $2500 \mathrm{~Hz}$ and $50 \%$ duty cycle). In that experiment, the absorption by DC was high because the total current by PDC was twice as strong.
Therefore, the absorption differed with investigators because the conditions of total current and voltage were not the same. Further investigation is needed under same conditions, i.e., an evaluation under constant voltage with constant current adjusted by application time.

\section{CONCLUSION}

The reduction in skin barrier function caused by DC and PDC were evaluated using the proposed method, which measures resistance in the short term. The application of PDC with around a $75 \%$ duty cycle was effective in reducing the barrier function without skin polarization. The sine waveform in addition to rectangular waveform (around 75\% duty cycle) decreased the barrier function more than other waveforms because the skin polarization and depolarization did not occur easily. The lower frequency (less than $100 \mathrm{~Hz}$ ) was effective in decreasing the barrier function, which contributed to the pulse length. The optimum conditions for the application of PDC, which decreased the skin barrier function without skin polarization, were a sine waveform or rectangular waveform (less than $100 \mathrm{~Hz}$ as frequency and around $75 \%$ as duty cycle). Under the conditions mentioned above, the absorption of drug would be expected to be enhanced.

\section{REFERENCES}

1) Kanebako M., Inagi T., Takayama K., Biol. Pharm. Bull., 25, 779782 (2002).

2) Kanebako M., Inagi T., Takayama K., STP Pharma. Sciences, submitted.

3) Kanebako M., Inagi T., Takayama K., Biol. Pharm. Bull., 25, 14561460 (2002).

4) Liu J. C., Sun Y., Siddiqui O., Chien Y. W., Shi W. M., Li J., Int. J. Pharmaceut., 44, 197-204 (1988).

5) Numajiri S., Sakurai H., Sugibayashi K., Morimoto Y., Omiya H., Takenaka H., Akiyama N., J. Pharm. Pharmcol., 45, 610-613 (1993).

6) Zakzewski C. A., Amory D. W., Jasaitis D. K., Li J. K.-J., Cardiovascular Drugs and Therapy, 6, 589-595 (1992).

7) Bagniefski T., Burnette R. R., J. Control. Release, 11, 113-122 (1990).

8) Preat V., Thysman S., Int. J. Pharmaceut., 96, 189-196 (1993).

9) Huang Y.-Y., Wu S.-M., Drug Dev. Ind. Pharm., 22, 1075-1081 (1996).

10) Knoblauch P., Moll F., J. Control. Release, 26, 203-212 (1993).

11) Chien Y. W., Lelawongs P., Siddiqui O., Sun Y., Shi W. M., J. Control. Release, 13, 263-278 (1990).

12) Santi P., Catellani P. L., Massimo G., Zanardi G., Colombo P., Int. J. Pharmaceut., 92, 23-28 (1993).

13) Cross S. E., Roberts M. S., J. Pharm. Sci., 84, 584-592 (1995).

14) Nakakura M., Kato Y., Hayakawa E., Kuroda T., Biol. Pharm. Bull., 19, 738-740 (1996).

15) Chen L.-L. H., Chien Y. W., J. Control. Release, 40, 187-198 (1996).

16) Chien Y. W., Siddiqui O., Shi W.-M., Lelawongs P., Liu J.-C., J. Pharm. Sci., 78, 376-383 (1989).

17) Chizmadzhev Y. A., Indenbom A. V., Kuzmin P. I., Galichenko S. V., Weaver J. C., Potts R. O., Biophys. J., 74, 843-856 (1998).

18) Hirvonen J., Hueber F., Guy R. H., J. Control. Release, 37, 239-249 (1995). 